\title{
SUR LA STRUCTURE DES COMPOSANTES CONNEXES SEMI-FREDHOLM DE $B(H)$
}

\author{
M. MBEKHTA
}

(Communicated by Palle E. T. Jorgensen)

\begin{abstract}
RÉsUMÉ. Dans ce travail, nous montrons que dans $B(H)$ les composantes connexes semi-Fredholm ont la même frontière et, d'autre part que $B(H)$ s'écrit comme la réunion de cette frontière commune $(\Delta)$ et de l'ensemble des opérateurs semi-Fredholm. Nous remarquons que $\Delta$ est un ensemble de première catégorie, stable pour les perturbations compactes.

Par ailleurs, nous montrons que l'intérieur de $\bar{B}(H)$, l'ensemble des opérateurs "réguliers", est exactement l'ensemble des opérateurs semi-Fredholm et que la frontière de $\bar{B}(H)$ est également $\Delta$.
\end{abstract}

\section{INTRODUCTION}

Soit $H$ un espace de Hilbert séparable et $B(H)$ l'algèbre des opérateurs bornés de $H$ dans lui-même. Pour $T \in B(H)$, notons $T^{*}, N(T)$ et $R(T)$ respectivement l'adjoint, le noyau et l'image de $T$.

$T$ est dit semi-Fredholm si $R(T)$ est fermé et $\min \left(\operatorname{dim} N(T), \operatorname{dim} N\left(T^{*}\right)\right)$ $<\infty$.

Notons, pour $T \in B(H)$, ind $(T)$, l'indice de $T$, définit par

$$
\operatorname{ind}(T)=\operatorname{dim} N(T)-\operatorname{dim} N\left(T^{*}\right)
$$

et si $\operatorname{dim} N(T)=\operatorname{dim} N\left(T^{*}\right)=\infty$ alors $\operatorname{ind}(T)=0$.

Soit $I_{n}=\{T \in B(H) ; \operatorname{ind}(T)=n\}$ avec $n \in \overline{\mathbf{Z}}=\mathbf{Z} \cup\{-\infty,+\infty\}$.

Soit $\mathscr{F}=\{T \in B(H) ; T$ semi-Fredholm $\}$, alors il est bien connu que $\mathscr{F}$ est un ouvert de $B(H)$ et que si on note $F_{n}=\{T \in \mathscr{F} ; \operatorname{ind}(T)=n\}$ alors $F_{n}$ est ouvert et connexe pour tout $n \in \overline{\mathbf{Z}}$.

Dans la première partie de ce travail, nous utilisons les résultats de Bouldin [1] et Izumino et Kato [4] sur l'adhérence de l'ensemble des opérateurs inversibles pour montrer que les composantes connexes semi-Fredholm $\left(F_{n}\right)$ ont la même frontière et que $B(H)$ s'écrit comme la réunion de cette frontière commune $(\Delta)$ et de $\mathscr{F}$ (Corollaire 1.4). Ceci montre qu'on peut imaginer $B(H)$ "comme" un cahier aux bords soudés et dont les feuilles seraient les composantes connexes semi-Fredholm. Nous remarquons aussi que $\Delta$ est un ensemble de première catégorie, stable pour les perturbations compactes.

Received by the editors January 29, 1991 and, in revised form, March 28, 1991.

1991 Mathematics Subject Classification. Primary 47A53, 47A55, 47B05.

Key words and phrases. Opérateur semi-Fredholm, régulier, frontière des composantes connexes semi-Fredholm. 
La deuxième partie de ce travail donne une réponse à une question posée par Harte (dans une communiation privée). $T$ est die "régulier" au sens de [3] si il existe $T^{\prime} \in B(H)$ tel que $T T^{\prime} T=T$. Nous montrons alors (Théorème 2.2) que l'intérieur de $\bar{B}(H)$, l'ensemble des opérateurs "réguliers" est exactement $\mathscr{F}$ et que la frontière de $\bar{B}(H)$ est également $\Delta$.

\section{Sur la structure des Composantes CONNEXES SEMI-FREDHOLM DE $\bar{B}(H)$}

Tout d'abord quelques notations:

$$
m_{e}(T)=\inf \left\{\sigma_{e}\left(T^{*} T\right)^{1 / 2}\right\}
$$

où $\sigma_{e}(T)$ est le spectre essentiel de $T$ définit par $\sigma_{e}(T)=\{\lambda \in \mathbf{C} ; T-\lambda I$ n'est pas Fredholm \}. Pour un ensemble $X$ de $B(H)$, on notera $\bar{X}$, int $X$, et $\partial X$ respectivement l'adhérence, l'intérieur, et la frontière de $X$.

$G$ désigne l'ensemble des opérateurs inversibles de $B(H)$.

Lemme 1.1 [1, Théorème 2]. (1) $m_{e}(T)>0 \Leftrightarrow R(T)$ fermé et $\operatorname{dim} N(T)<\infty$;

(2) $m_{e}\left(T^{*}\right)>0 \Leftrightarrow \Omega(T)$ fermé et $\operatorname{dim} N\left(T^{*}\right)<\infty$.

Théorème $1.2[4$, Théorème 4.1]. $\forall n \in \bar{Z}$

(1) si $T \in I_{n}$ alors $\operatorname{dist}\left(T, F_{n}\right)=0$;

(2) si $T \notin I_{n}$ alors $\operatorname{dist}\left(T, F_{n}\right)=\max \left\{m_{e}(T), m_{e}\left(T^{*}\right)\right\}$.

Théorème 1.3. $T \notin \bar{F}_{j} \Leftrightarrow T \in \bigcup_{k \neq j} F_{k}$.

Démonstration. $(\Rightarrow)$ Si $T \notin \bar{F}_{j}$ alors $\operatorname{dist}\left(T, F_{j}\right)>0$ et $T \notin I_{j}$. D'après le Théorème 1.2(2), $\operatorname{dist}\left(T, F_{j}\right)=\max \left\{m_{e}(T), m_{e}\left(T^{*}\right)\right\}$ d'où $m_{e}(T)>0$ ou $m_{e}\left(T^{*}\right)>0$, le Lemme 1.1 implique que $T \in \bigcup_{k \neq j} F_{k}$.

$(\Leftarrow)$ Si $T \in \bigcup_{k \neq j} F_{k}$ alors il existe $k \neq j$ tel que $T \in F_{k}$. Puisque $F_{k}$ est ouvert, on voit que $\operatorname{dist}\left(T, F_{j}\right)>0$ et, par conséquent, $T \notin \bar{F}_{j}$.

Corollaire 1.4. La frontière de $\bar{F}_{j}\left(\partial \bar{F}_{j}\right)$ ne dépend pas de $j$ et si on note $\Delta=\partial \bar{F}_{j}$ alors $\Delta=\partial F_{j}$ et

$$
B(H)=\Delta \cup\left[\bigcup_{k} F_{k}\right] .
$$

Démonstration. Montrons d'abord que int $\bar{F}_{j}=F_{j}$ pour tout $j$. L'inclusion " $F_{j} \subseteq$ int $\bar{F}_{j}$ " étant évident, il suffit de montrer l'autre inclusion.

En effet, pour $j=0$, le résultat se déduit de [4, Corollaire 2.2(1)]. Soit $j \neq 0$ et $T \in \operatorname{int} \bar{F}_{j}$, d'après le théorème précédent, $T \notin \bigcup_{k \neq j} F_{k}$ donc si $T$ est semi-Fredholm alors $T \in F_{j}$. Supposons que $T$ n'est pas semi-Fredholm alors $m_{e}(T)=m_{e}\left(T^{*}\right)=0$ d'où $T \in \partial \bar{G}=\partial F_{0}$ (voir [4, Théorème 2.1 et Corollaire 2.2(2)]).

Donc $T \in \operatorname{int} \bar{F}_{j} \cap \partial F_{0}$ ce que entraîne que $\bar{F}_{j} \cap F_{0} \neq \varnothing$ contradiction (continuité de l'indice).

Remarquons maintenant, que d'après le théorème précédent, on a $\forall j, B(H)$ $=\bar{F}_{j} \cup\left[\bigcup_{k \neq j} F_{k}\right]$ d'où $B(H)=\partial \bar{F}_{j} \cup F_{j} \cup\left[\bigcup_{k \neq j} F_{k}\right]$ (puisque int $\bar{F}_{j}=F_{j}$ ) donc $B(H)=\partial \bar{F}_{j} \cup\left[\bigcup_{k} F_{k}\right]=\partial F_{j} \cup\left[\bigcup_{k} F_{k}\right]$ et comme $\forall k \neq j, \partial \bar{F}_{j} \cap F_{k}=\varnothing$ 
(continuité de l'indice), on en déduit que $\partial \bar{F}_{j}=\partial F_{j}$ ne dépend pas de $j$ et, par conséquent, si on note $\Delta=\partial \bar{F}_{j}$ alors $B(H)=\Delta \cup\left[\bigcup_{k} F_{k}\right]$ et le corollaire est démontré.

Corollaire 1.5. Soit $T \in B(H)$ avec $R(T)$ fermé, $j \in \bar{Z}$. Alors les conditions suivantes sont équivalents

(1) $T \in \partial \bar{G}$;

(2) $T \in \partial \bar{F}_{j}=\partial F_{j}$;

(3) $\operatorname{dim} N(T)=\operatorname{dim} N\left(T^{*}\right)=\infty$.

Démonstration. Conséquence directe du corollaire précédent.

Remarque. (1) $\Delta$ est un fermé avec intérieur vide (i.e. de première catégorie);

(2) $\Delta$ est stable pour les perturbations compactes $(\Delta+\mathscr{K}(H)=\Delta$, où $\Delta+$ $\mathscr{K}(H)=\{T+S ; T \in \Delta$ et $S$ compact $\}$ ). en effet si $T \in \Delta$ et $S$ compact et si $T+S \notin \Delta$ alors il est facile de voir que $T \in \mathscr{F}$, contradiction.

2. Caractérisation de L'intérieur des opérateurs "RÉguliers"

Notons $B(H)$, l'ensemble des opérateurs "réguliers" au sens de [3], par définition

$$
\bar{B}(H)=\left\{T \in B(H) ; \exists T^{\prime} \in B(H) \text { tel que } T T^{\prime} T=T\right\},
$$

$T^{\prime}$ est appelé inverse généralisé de $T$. On remarquera, qu'on peut normaliser $T^{\prime}$, par $T^{\prime \prime}=T^{\prime} T T^{\prime}$, alors $T T^{\prime \prime} T=T$ et $T^{\prime \prime} T T^{\prime \prime}=T^{\prime \prime}$.

Tout d'abord, on a le lemme suivant

Lemme 2.1. Soit $T \in \bar{B}(H)$ alors $T \in B(H) \Leftrightarrow R(T)$ fermé.

Démonstration. $(\Rightarrow)$ Supposons que $T T^{\prime} T=T$, alors $T T^{\prime}$ est une projection sur $R(T)$, continue. Donc $R(T)$ est fermé.

$(\Leftarrow)$ Si $R(T)$ est fermé, alors la restriction de $T, \widehat{T}: N(T)^{\perp} \rightarrow R(T)$ est inversible.

Posons:

$$
T^{\prime}= \begin{cases}\widehat{T}^{-1} & \text { sur } R(T) \\ 0 & \text { sur } R(T)^{\perp}\end{cases}
$$

alors $T^{\prime}$ est un opérateur fermé et a pour domaine $R(T) \oplus R(T)^{\perp}=H$. Donc $T^{\prime} \in B(H)$. Et, il est facile de voir que $T T^{\prime} T=T$.

Remarque. $\mathscr{F} \subseteq \bar{B}(H)$.

Théorème 2.2. int $B(H)=\mathscr{F}$.

Démonstration. D'après la remarque précédent et puisque $\mathscr{F}$ est ouvert, il suffit de montrer que $\operatorname{int} \overparen{B}(H) \subseteq \mathscr{F}$. Soit $T \in \operatorname{int} \mathscr{B}(H)$. Supposons que $\operatorname{dim} N(T)=\operatorname{dim} N\left(T^{*}\right)=\infty$ et soit $e_{1}, e_{2}, \ldots, e_{n}, \ldots$ une suite orthonormale d'éléments de $N(T)$ et $f_{1}, f_{2}, \ldots, f_{n}, \ldots$ une suite orthonormale d'éléments de $N\left(T^{*}\right)$.

Posons $H_{1}=$ c.l.m. $\left\{e_{1}, e_{2}, \ldots, e_{n}, \ldots\right\}$ et $H_{2}=$ c.l.m. $\left\{f_{1}, f_{2}, \ldots, f_{n}, \ldots\right\}$ (avec la possibilité, $H_{1}=N(T)$ et $H_{2}=N\left(T^{*}\right)$ ).

Soit $S_{0}$ l'application de $H_{1}$ dans $H_{2}$ définie par $S_{0} e_{i}=\frac{1}{i} f_{i}$ et

$$
S u= \begin{cases}S_{0} u & \text { si } u \in H_{1}, \\ 0 & \text { si } u \in H_{1}^{\perp}\end{cases}
$$


alors $\forall \varepsilon>0, R(T+\varepsilon S)$ n'est pas fermé et, par conséquent (Lemme 2.1), $T+$ $\varepsilon S \notin \vec{B}(H)$ et $\|T-(T+\varepsilon S)\|=\|\varepsilon S\| \leq \varepsilon$, contradiction car $T \in \operatorname{int} B(H)$.

Corollaire 2.3. Soit $T \in \operatorname{int} B(H)$ alors $\exists \delta>0$ et $T_{\lambda}^{\prime}:\{|\lambda|<\delta\} \rightarrow B(H)$ méromorphe tel que

$$
(T-\lambda) T_{\lambda}^{\prime}(T-\lambda)=T-\lambda \text { pour tout }|\lambda|<\delta .
$$

Démonstration. D'après le théorème précédent, si $T \in$ int $B(H)$ alors $T$ est semi-Fredholm, d'où en utilisant [5, Théorème 4], on en déduit qu'il existe $M$, $N$ deux sous-espaces fermés de $H$, invariants par $T$ tels que $T_{\mid \mathbf{N}}$ est nilpotent de degré $d \in \mathbf{N}$ fini et si on note $T_{0}=T_{\mid M}$ alors $\forall n \geq 0, N\left(T_{0}^{n}\right) \subseteq R\left(T_{0}\right)$, $R\left(T_{0}\right)$ étant fermé. Par conséquent [6, Théorème 2.6] implique qu'il existe $\delta>0$ et $B_{\lambda}:\{|\lambda|<\delta\} \rightarrow B(M)$ analytique tel que $\left(T_{0}-\lambda\right) B_{\lambda}\left(T_{0}-\lambda\right)=T_{0}-\lambda$ pour tout $|\lambda|<\delta$.

Si $N=\{0\}$ (i.e., $M=H$ ) alors $T=T_{0}$, on pose $T_{\lambda}^{\prime}=B_{\lambda}$. Sinon, $T_{\mid \mathbf{N}}$ nilpotent de degré $d$ implique que $\forall \lambda \neq 0,\left(T_{\mid \mathbf{N}}-\lambda\right)$ est inversible dans $B(N)$.

Posons $T_{\lambda}^{\prime}=B_{\lambda} \oplus\left(T_{\mid \mathbf{N}}-\lambda\right)^{-1}$ pour $0<|\lambda|<\delta$ et $T_{0}^{\prime}$ un inverse généralisé de $T(T \in \overparen{B}(H))$, alors $T_{\lambda}^{\prime}$ ainsi défini est analytique dans $0<|\lambda|<\delta$ avec $\lambda=0$ comme pôle d'ordre $d$ (puisque $T_{\mid \mathbf{N}}$ est nilpotent de degré $d$ ) et il est clair que $(T-\lambda) T_{\lambda}^{\prime}(T-\lambda)=T_{\lambda}$ pur tout $|\lambda|<\delta$.

Corollaire 2.4. $\partial \vec{B}(H)=\Delta$.

Démonstration. Soit $\mathscr{M}=\{T \in B(H) ; T$ inversible à gauche ou à droite $\}$. Il est clair que $\mathscr{M} \subseteq B(H)$. D'après [2, Problème 140], $\mathscr{M}$ est dense dans $B(H)$ et donc $\mathbb{B}(H)$ est dense dans $B(H)$ et, par conséquent, $\partial \vec{B}(H)=$ $B(H) \backslash$ int $B(H)=B(H) \backslash \mathscr{F}=\Delta$ (voir Corollaire 1.4).

\section{RÉFÉRENCES}

1. R. Bouldin, The essential minimum modulus, Indiana Univ. Math. J. 30 (1981), 513-517.

2. P. R. Halmos, A Hilbert space problem book, 2eme éd., Van Nostrand, Princeton, NJ, 1980.

3. R. E. Harte, Invertibility and singularity, Marcel Dekker, New York, 1988.

4. S. Izumino and Y. Kato, The closure of invertible operators on Hilbert space, Acta. Sci. Math. 49 (1985), 321-327.

5. T. Kato, Perturbation theory for nullity, deficiency and other quantities of linear operators, J. Analyse Math. 6 (1958), 261-322.

6. M. Mbekhta, Généralisation de la décomposition de Kato aux opérateurs paranormaux et spectraux, Glasgow Math. J. 29 (1987), 159-175.

Université des Sciences et Techniques de lille Flandres Artois, U. F. R. de Mathematiques Pures et Appliquées, U.R.A C.N.R.S. 751, 59655-Villeneuve D'AscQ CedeX, FRANCE 